intratumoral TLR3 expression and T-cell infiltration is currently analyzed.

Results Global deficiency of murine Tlr3, or TRIF, induced significantly increased digestive tumor formation, associated with increased morbidity indicating a tumor suppressive role. Coherently, TLR3 expression is highly significantly decreased in human colorectal cancer compared to normal mucosa, significantly correlated with poor survival. TLR3 deficient cell lines show reduced migration and slightly declined proliferation suggesting an oncogenic role on the cell-autonomous level. Nevertheless, gene expression analysis revealed that the dsRNA induced expression of T-cell attracting cytokines CXCL10 and CXCL11 in colon cancer cell lines is exclusively dependent on TLR3. These chemokines were shown to favor a TH1-type antitumoral response.

Conclusions TLR3 favors tumor suppression in vivo, presumably resulting from non-cell-autonomous factors such as the production of CXCL10 and 11 and resulting T-cell infiltration. This may outweigh the putative cell-autonomous oncogenic functions of TLR3 deficiency.

Disclosure Information A. Sichler: None. M. Frey: None. W. Johannes: None. K. Janssen: None.

\section{P09.12 BIFUNCTIONAL SIRP $\alpha$-CD123 FUSION ANTIBODY FOR THE ELIMINATION OF ACUTE MYELOID LEUKEMIA STEM CELLS}

${ }^{1} S$ Tahk*, 'SM Schmitt, ${ }^{2,3}$ B Vick, ${ }^{4} \mathrm{C}$ Augsberger, 'L Pascual Ponce, 2,3,5! Jeremias, ${ }^{6} \mathrm{G}$ Wittmann, ${ }^{4,3,7} \mathrm{M}$ Subklewe, ${ }^{1} \mathrm{NC}$ Fenn, ${ }^{1} \mathrm{~K}$ Hopfner. ${ }^{1}$ Department of Biochemistry and Gene Center, Ludwig-Maximilians-Universität München, Munich, Germany; ${ }^{2}$ Research Unit Apoptosis in Hematopoietic Stem Cells, Helmholtz Zentrum München, German Research Center for Environmental Health, Munich, Germany; ${ }^{3}$ German Cancer Consortium (DKTK) and German Cancer Research Center (DKFZ), partner site Munich, Heidelberg, Germany; ${ }^{4}$ Laboratory of Translational Cancer Immunology, Ludwig-Maximilians-Universität München, Munich, Germany; ${ }^{5}$ Department of Oncology, Dr von Haunersches Kinderspital, Ludwig Maximilians-Universität München, Munich, Germany; ${ }^{6}$ Department of Transfusion Medicine, University Hospital, Ludwig-Maximilians-Universität München, Munich, Germany; ${ }^{7}$ Department of Hematology and Oncology, Medizinische Klinik und Poliklinik III, Klinikum der Universität München, Munich, Germany

\subsection{6/jitc-2020-ITOC7.112}

Background Despite advances in the development of novel strategies against acute myeloid leukemia (AML), treatment options are limited and most patients relapse. Relapse occurs due to the persistence of chemotherapy-resistant leukemic stem cells (LSCs), which re-initiate the outgrowth of the disease, highlighting the need of targeting LSCs to improve patient survival. LSCs are characterized by the expression of the interleukin-3 receptor $\alpha$, also known as CD123. CD123 is expressed on AML blasts and LSCs, and shows a moderate expression on normal hematopoietic stem cells, claiming CD123 as a suitable target antigen. CD47 is a ubiquitously expressed immune checkpoint upregulated on LSCs where it acts as an immune escape mechanism. CD47 transmits a 'don't eat me' signal upon its interaction with the signal regulatory protein alpha (SIRP $\alpha$ ) receptor on macrophages, thus inhibiting phagocytosis. In order to efficiently eliminate LSCs, we have designed a bifunctional antibody that specifically targets CD123 and simultaneously blocks CD47. Importantly, our strategy restricts the benefits of the CD47 blockade to $\mathrm{CD}_{123^{+}}$AML cells. Thus, we hypothesize a lower risk for on-target off-leukemia toxicity.
Materials and Methods The bifunctional SIRPo-CD123 antibody was generated by fusing an extracellular domain of the SIRP $\alpha$ receptor, which functions as the CD47 blocking domain, to the CD123 antibody. The biological activity of the SIRP $\alpha$-CD123 antibody was examined using AML-derived MOLM-13 cells, primary AML patient material and patientderived xenografted (PDX) AML cells with NOD.Cg-Prkdc ${ }^{\text {scic }}$ IL2 $\mathrm{rg}^{\mathrm{tm} 1 \mathrm{Wjl}} / \mathrm{SzJ}$ (NSG) mice.

Results The SIRP $\alpha$ fusion improved the binding of the bifunctional SIRPQ-CD123 antibody to AML cells compared to a conventional CD123 antibody. The SIRP $\alpha$-CD123 antibody enhanced the elimination of the AML-derived MOLM-13 cells by antibody-dependent cellular cytotoxicity via NK cells. Additionally, the cytotoxicity was confirmed using primary patientderived AML cells. Furthermore, an improved cytotoxicity towards leukemia initiating AML PDX cells was observed with the SIRP $\alpha$-CD123 antibody using luciferase bioluminescence in vivo imaging. With regards to the inhibition of CD47 signaling, we were able to show a blockade of CD47 on $\mathrm{CD} 123{ }^{+} \mathrm{CD} 47^{+}$cells by the SIRPo-CD123 antibody. Correspondingly, a significant increase in phagocytosis of primary patient-derived AML cells mediated by monocyte-derived macrophages was observed in both allogenic and autologous setting. We were also able to show a preferential binding to MOLM-13 in the presence of a 20-fold excess of red blood cells indicating a potential low on-target off-leukemia toxicity.

Conclusions The bifunctional SIRP $\alpha$-CD123 fusion antibodies target the CD123+CD47+ cells and stimulate their phagocytosis by blocking the inhibitory CD47 signal. The dual mode of action of the SIRP $\alpha-C D 123$ has the potential to deplete the AML LSCs through NK cell cytotoxicity and macrophagemediated phagocytosis while restricting the CD47 related ontarget off-leukemia toxicity.

Support H2020-EU grant agreement no 641549

Disclosure Information S. Tahk: None. S.M. Schmitt: None. B. Vick: None. C. Augsberger: None. L. Pascual Ponce: None. I. Jeremias: None. G. Wittmann: None. M. Subklewe: None. N. C. Fenn: None. K. Hopfner: None.

\section{P09.13 OPTIMIZATION OF A GMP-GRADE LARGE-SCALE EXPANSION PROTOCOL FOR CYTOKINE-INDUCED KILLER CELLS USING GAS-PERMEABLE STATIC CULTURE FLASKS}

${ }^{1} \mathrm{~A}$ Ventura* ${ }^{1} \mathrm{P}$ Palmerini, ${ }^{1} \mathrm{~A}$ Dalla Pietà, ${ }^{2} \mathrm{R}$ Sommaggio, ${ }^{3} \mathrm{G}$ Astori, ${ }^{3} \mathrm{~K}$ Chieregato, ${ }^{4} \mathrm{M}$ Tisi ${ }^{5} \mathrm{C}$ Visco, ${ }^{4} \mathrm{O}$ Perbellini, ${ }^{4} \mathrm{M}$ Ruggeri, ${ }^{1} \mathrm{E}$ Cappuzzello, ${ }^{1,2} \mathrm{~A}$ Rosato. ${ }^{1}$ University of Padua, Padua, Italy; ${ }^{2}$ Veneto Institute of Oncology IOV - IRCCS, Padua, Italy; ${ }^{3}$ Advanced Cellular Therapy Laboratory, Department of Hematology, Vicenza Hospital, Vicenza, Italy ${ }^{4}$ Hematology Department, San Bortolo Hospital, Vicenza, Italy; ${ }^{5}$ Department of Medicine, Section of Hematology, University of Verona, Verona, Italy

\subsection{6/jitc-2020-ITOC7.113}

Background Cytokine-Induced Killer (CIK) cells are ex vivo expanded T cells with NK cell phenotype. They express both CD3 and CD56 antigens, and exert a potent antitumor activity against a variety of tumors. Several clinical trials demonstrated the safety and the feasibility of CIK cell therapy, with very low side effects and minimal graft-versus-host toxicity. In this study, we developed a GMP-compliant protocol for robust large-scale expansion of CIK cells using G-Rex ${ }^{\circledR}$ gas-permeable static culture flasks.

Materials and Methods CIK cells were obtained by stimulating healthy donor PBMCs with GMP-grade IFN- $\gamma$, IL-2 and CD3 $\mathrm{mAbs}$, and were cultured in G-Rex $6^{\circledR}$ or G-Rex ${ }^{\circledR} 6 \mathrm{M}$ well 